\title{
Acute Spontaneously Resolving Pulmonary Vasculitis: A Case Report
}

\author{
James B. Geake ${ }^{1}$ and Graeme Maguire ${ }^{2,3}$ \\ ${ }^{1}$ Department of Respiratory and Sleep Medicine, Monash Medical Centre, Melbourne, VIC 3206, Australia \\ ${ }^{2}$ Cairns Clinical School, School of Medicine and Dentistry, Faculty of Medicine, Health and Molecular Sciences, James Cook University, \\ P.O. Box 902, Cairns, QLD 4870, Australia \\ ${ }^{3}$ Baker IDI Central Australia, P.O. Box 1294, Alice Springs, NT 0870, Australia \\ Correspondence should be addressed to James B. Geake, james.geake@southernhealth.org.au
}

Received 31 August 2012; Accepted 24 October 2012

Academic Editors: A. M. Mansour and A. V. Vanikar

Copyright () 2012 J. B. Geake and G. Maguire. This is an open access article distributed under the Creative Commons Attribution License, which permits unrestricted use, distribution, and reproduction in any medium, provided the original work is properly cited.

This is the first description that we are aware of describing the spontaneous resolution of an acute pulmonary vasculitis, possibly secondary to microscopic polyangiitis. Haemoptysis is a common symptom for patients presenting to primary and tertiary referral centres, and pulmonary vasculitis is one of a variety of aetiologies that should always be considered. The pulmonary vasculitides are difficult diagnostic and management problems. They are encumbered by a relative paucity of level 1 evidence addressing their diagnosis, classification, and treatment. This is therefore an important paper to publish because it adds to the global breadth of experience with this important clinical condition.

In July 2010 a 43 -year-old female presented with a $24-$ hour history of $30 \mathrm{~mL}$ of frank haemoptysis. There were no symptoms suggestive of a respiratory infection. She worked as an office manager and had no relevant occupational or environmental exposures. She had ceased smoking ten years previously having accumulated a ten-pack-year history. She had hypertension treated with amlodipine and perimenstrual migraines. Both parents and her brother were alive without any significant medical diseases.

On presentation peripheral arterial oxygen saturation was $85 \%$ on room air, respiratory rate was 24 per minute, blood pressure was 200/100, and temperature was $38.5^{\circ} \mathrm{C}$. She remained afebrile thereafter. An ECG demonstrated sinus rhythm, 100 beats per minute. A chest Xray demonstrated bilateral perihilar infiltrates. A CTPA confirmed diffuse bilateral alveolar infiltrates (Figure 1). No vascular filling defects were identified. Haemoglobin was $99 \mathrm{~g} / \mathrm{L}$ (115-160). MCV (mean cell volume) was $67 \mathrm{fL}$ (81-96), and the film was suggestive of iron deficiency. White cell count was mildly elevated at $137 / \mathrm{nL}(3.5-11)$. Other inflammatory markers were also mildly elevated. ESR (erythrocyte sedimentation rate) was $29 \mathrm{~mm}(<15)$.
CRP (C-reactive protein) was $68.1 \mathrm{mgl} / \mathrm{L}(<8)$. Creatinine was $65 \mu \mathrm{mol} / \mathrm{L}(50-100)$. Urea was $48 \mu \mathrm{mol} / \mathrm{L}(2.7-7.8)$. Electrolytes were within normal limits. INR (international normalised ratio) was $1.1(0 \cdot 9-1.2)$. APTT (activated partial thromboplastin time) was 28 seconds (25-34). Testing for antiglomerular basement membrane, antiuclear cytoplasmic antibodies (cANCA and pANCA), antinuclear antibodies, and extractable nuclear antigens (ENA) were all negative. Rheumatoid factor was $<10 \mathrm{IU} / \mathrm{mL}(<30)$. Several sputum samples were sent. No pathogens were identified on standard bacterial and mycobacterial cultures. There was no cytological evidence of malignancy. Urinalysis was normal. Phase contrast microscopy was not performed as the red cell count on several specimens was within normal limits. Albumin/creatinine ratio was $17.7 \mathrm{~g} / \mathrm{mol}$ creatinine $(<3.5)$. The patient was treated with benzylpenicillin and doxycycline, and there was improved, but persisting haemoptysis. On the third day the patient proceeded to fibre optic bronchoscopy where no source of bleeding or endobronchial abnormality was identified. A surgical lung biopsy was subsequently performed. At the operation blood was noted within the pleural space, the surface of the lung was oozing 


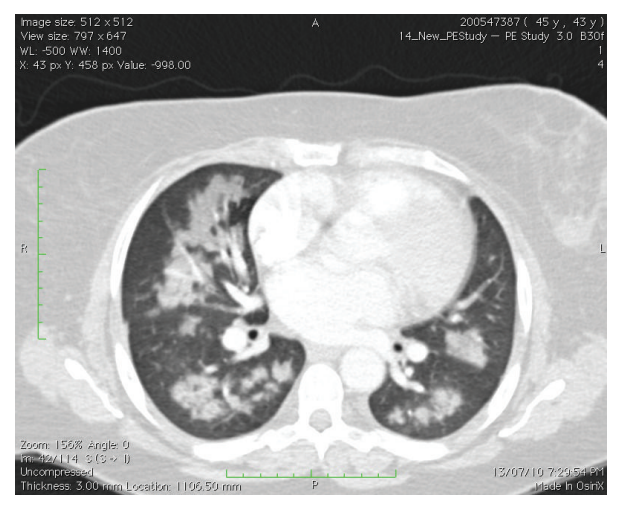

FIgURE 1: Coronal CT chest demonstrating bilateral perihilar pulmonary infiltrates consistent with diffuse alveolar haemorrhage.

fresh blood, and the parenchyma was boggy. A wedge biopsy was taken from the right middle lobe. Microscopy demonstrated a widespread interstitial inflammatory process centred on capillary sized vessels. There were associated interstitial haemorrhage and fibrin deposition. Occasional neutrophils and eosinophils were both observed. The disease process was variable in intensity with propensity for different expression on either side of an interlobular septum. No granulomata were identified. Special stains were negative for fungal hyphae and Pneumocystis species (Figures 2 and 3). A diagnosis of small vessel pulmonary vasculitis was made, thought to be most consistent with microscopic polyangiitis.

The patient made an uneventful recovery from the surgical procedure and the haemoptysis appeared to rapidly spontaneously settle. Induction immunosuppression with cyclophosphamide and high dose prednisolone was discussed. However, the patient declined. The patient was therefore, discharged three days after the operation with close outpatient surveillance arranged. Repeated clinical reviews with an assessment of lung function, chest radiology, renal function, and urinalysis demonstrated continued complete resolution of pulmonary vasculitis. 12 months after her initial presentation she remained well.

Pulmonary vasculitis should be considered in the differential diagnosis of haemoptysis. Microscopic polyangiitis is a small vessel systemic vasculitis that usually manifests with glomerulonephritis. The lungs are involved in association in approximately $20 \%$ to $30 \%$ of cases, with diffuse alveolar haemorrhage in approximately $10 \%$ to $20 \%[1,2]$. It is associated with pANCA in $90 \%$ of the cases [3]. It is rare for this disease to present with diffuse alveolar haemorrhage in isolation. Treatment recommendations are largely based on historical data demonstrating poor prognosis for patients with pauciimmune glomerulonephritis. No randomised controlled trials have been performed for patients with pulmonary haemorrhage. Many advocate aggressive immunosuppression in this clinical situation with a combination of cyclophosphamide and high dose of prednisolone, with or without plasma exchange [4]. Owing to the toxic effects of this regimen, different strategies have been investigated, and promising disease modifying and biological alternatives

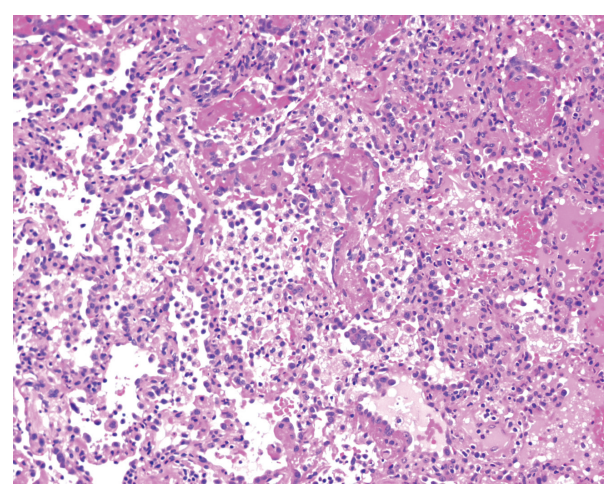

Figure 2: Low power magnification of hematoxylin and eosin stained lung biopsy demonstrating a pauci-immune small vessel vasculitis.

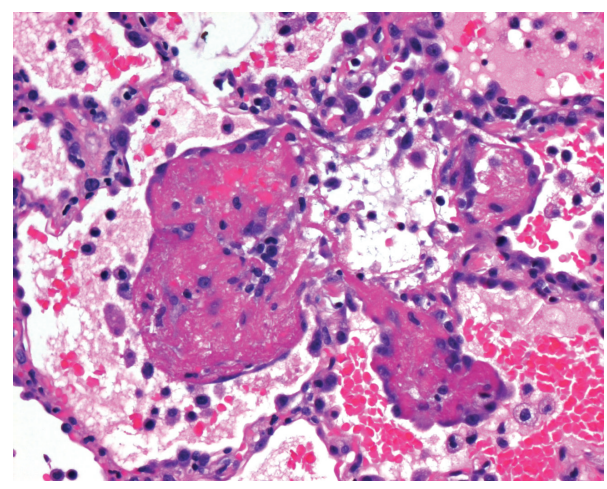

FIGURE 3: High power magnification of hematoxylin and eosin stained lung biopsy demonstrating a pauci-immune small vessel vasculitis.

continue to emerge, both for induction and maintenance immunosuppression.

Here we described the unusual case of small vessel pulmonary vasculitis with spontaneous remission. Whilst the histology is thought to favour a diagnosis of microscopic polyangiitis, it is possible that an alternate diagnosis was missed, for example, through sampling error. This case highlights the difficulties applying guidelines to rare diseases, particularly when presentations are atypical, as was the case of this patient.
Abbreviations
MCV: Mean cell volume
ESR: Erythrocyte sedimentation rate
CRP: C-reactive protein
INR: International normalised ratio
ENA: Extractable nuclear antigens
ANCA: Antinuclear cytoplasmic antibodies. 


\section{Conflict of Interests}

Both James Geake and Graeme Maguire confirm there were no financial or other sources of support in the preparation of this paper, and there are no conflicts of interests.

\section{Acknowledgments}

There were no internal or external funding sources. J. Geake was responsible for the review of the case data, drafting of the paper, and the review of the literature. G. Maguire was responsible for the supervision and editing of the final paper. Both are guaranators for the final paper. The patient described in this case report was treated by the authors at the Royal Hobart Hospital, in Tasmania, Australia.

\section{References}

[1] C. Agard, L. Mouthon, A. Mahr, and L. Guillevin, "Microscopic polyangiitis and polyarteritis nodosa: how and when do they start?" Arthritis Care and Research, vol. 49, no. 5, pp. 709-715, 2003.

[2] S. E. Lane, R. A. Watts, L. Shepstone, and D. G. I. Scott, "Primary systemic vasculitis: clinical features and mortality," QJM, vol. 98, no. 2, pp. 97-111, 2005.

[3] L. Guillevin, B. Durand-Gasselin, R. Cevallos et al., "Microscopic polyangiitis: clinical and laboratory findings in 85 patients," Arthritis \& Rheumatism, vol. 42, pp. 421-430, 1999.

[4] P. J. Klemmer, W. Chalermskulrat, M. S. Reif, S. L. Hogan, D. C. Henke, and R. J. Falk, "Plasmapheresis therapy for diffuse alveolar haemorrhage in patients with small-vessel vasculitis," American Journal of Kidney Diseases, vol. 42, no. 6, pp. 11491153, 2003. 


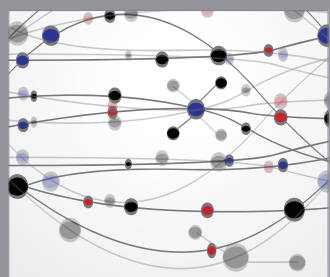

The Scientific World Journal
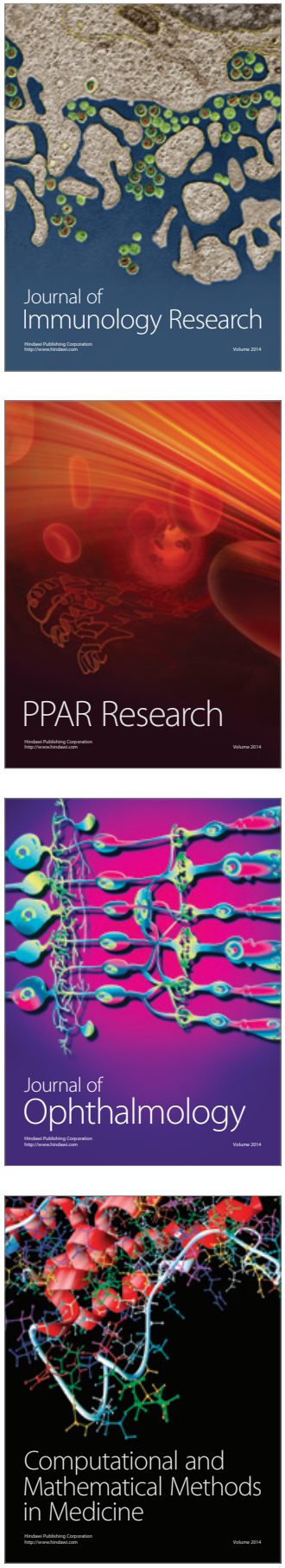

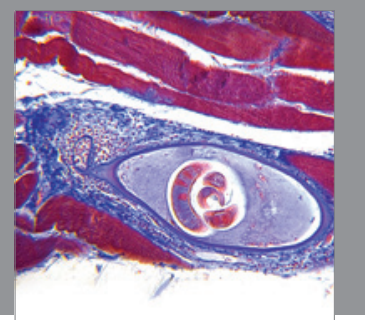

Gastroenterology

Research and Practice
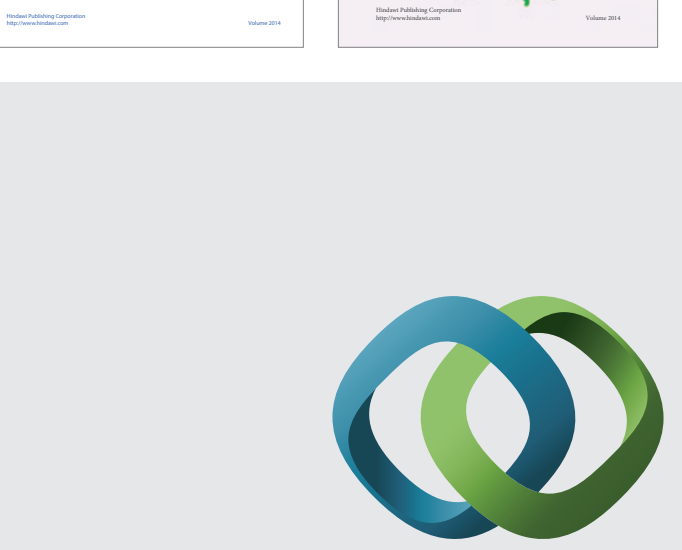

\section{Hindawi}

Submit your manuscripts at

http://www.hindawi.com
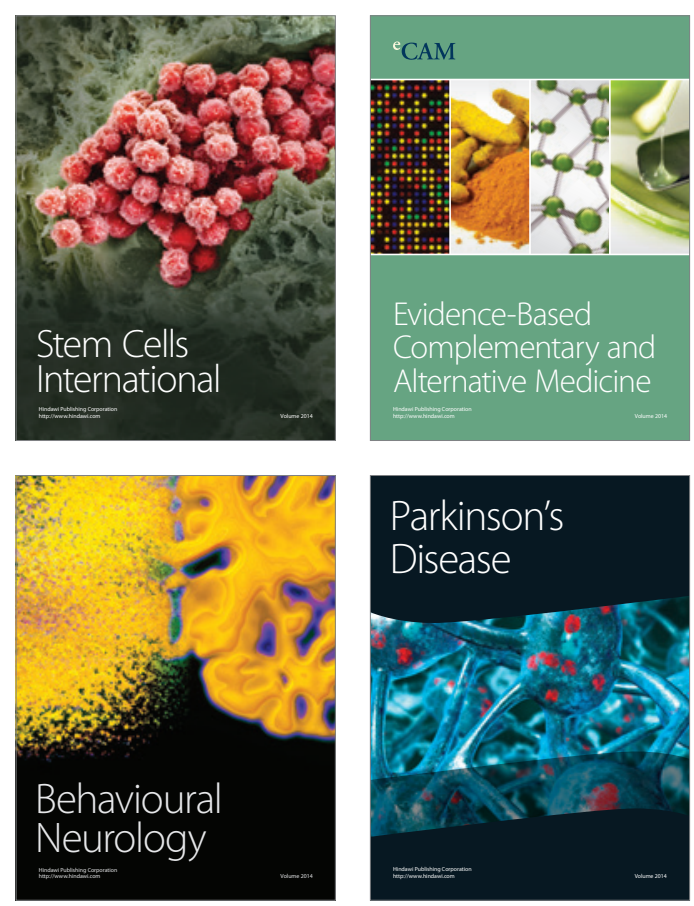

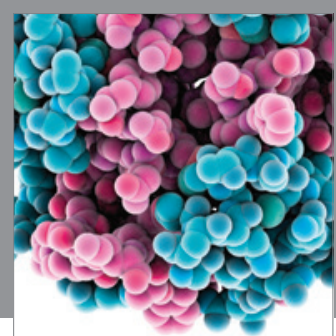

Journal of
Diabetes Research

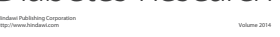

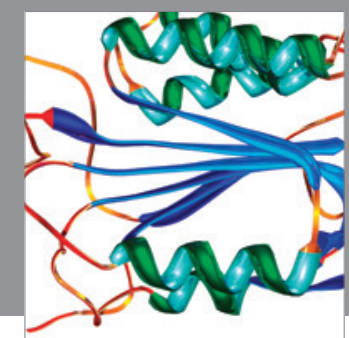

Disease Markers
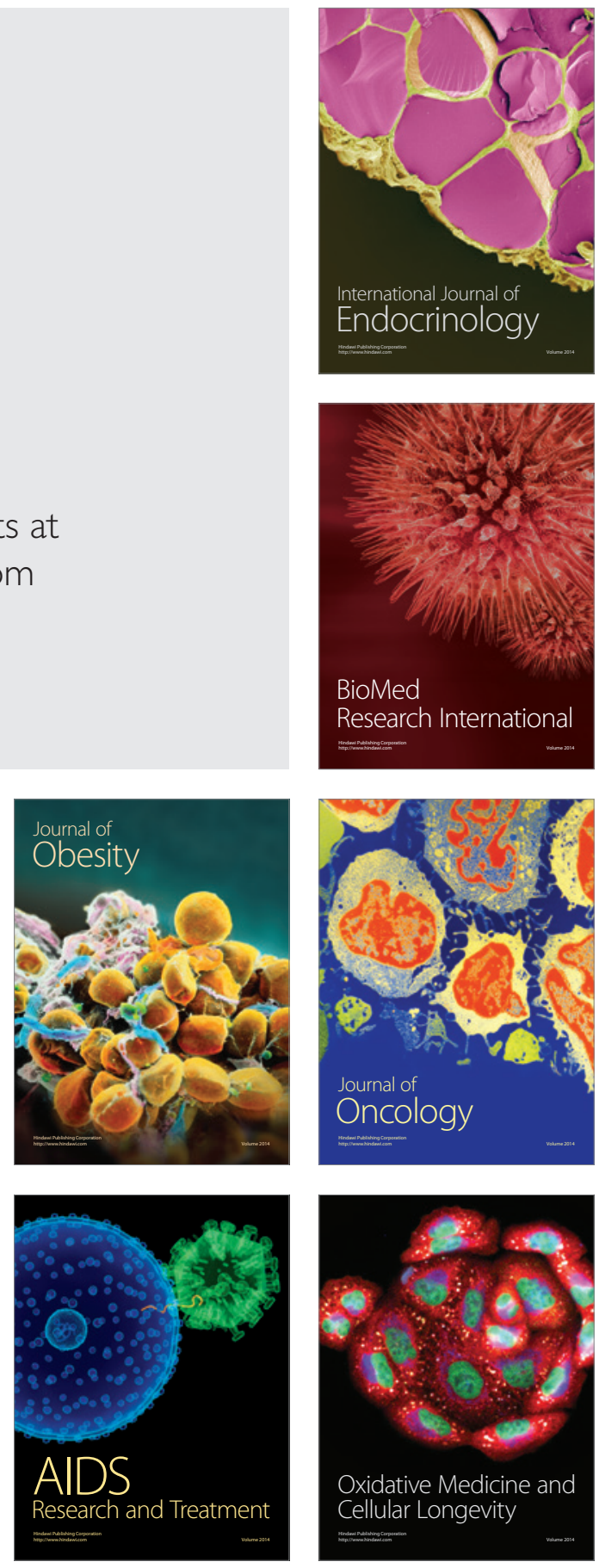